\title{
Phylogenetic analysis of faecal microbiota from captive cheetahs reveals underrepresentation of Bacteroidetes and Bifidobacteriaceae
}

\author{
Anne AMJ Becker ${ }^{1,2^{*}}$, Myriam Hesta ${ }^{2}$, Joke Hollants ${ }^{1}$, Geert PJ Janssens ${ }^{2}$ and Geert Huys ${ }^{1,3}$
}

\begin{abstract}
Background: Imbalanced feeding regimes may initiate gastrointestinal and metabolic diseases in endangered felids kept in captivity such as cheetahs. Given the crucial role of the host's intestinal microbiota in feed fermentation and health maintenance, a better understanding of the cheetah's intestinal ecosystem is essential for improvement of current feeding strategies. We determined the phylogenetic diversity of the faecal microbiota of the only two cheetahs housed in an EAZA associated zoo in Flanders, Belgium, to gain first insights in the relative distribution, identity and potential role of the major community members.

Results: Taxonomic analysis of 165 rRNA gene clone libraries (702 clones) revealed a microbiota dominated by Firmicutes (94.7\%), followed by a minority of Actinobacteria (4.3\%), Proteobacteria (0.4\%) and Fusobacteria (0.6\%). In the Firmicutes, the majority of the phylotypes within the Clostridiales were assigned to Clostridium clusters XIVa (43\%), XI (38\%) and I (13\%). Members of the Bacteroidetes phylum and Bifidobacteriaceae, two groups that can positively contribute in maintaining intestinal homeostasis, were absent in the clone libraries and detected in only marginal to low levels in real-time PCR analyses.

Conclusions: This marked underrepresentation is in contrast to data previously reported in domestic cats where Bacteroidetes and Bifidobacteriaceae are common residents of the faecal microbiota. Next to methodological differences, these findings may also reflect the apparent differences in dietary habits of both felid species. Thus, our results question the role of the domestic cat as the best available model for nutritional intervention studies in endangered exotic felids.
\end{abstract}

Keywords: Bacterial community sequencing, Exotic felids, Wildlife conservation, Zoo nutrition

\section{Background}

In the broad scope of wildlife conservation with the aim to protect animal species from extinction, researchers and zoo managers face significant challenges in the conservation of threatened and endangered species. In zoo animal husbandry, nutrition is one of the most critical components [1]. Feeding mismanagement may give rise to suboptimal health, low breeding performance and a higher incidence of gastrointestinal and metabolic

\footnotetext{
* Correspondence: anne.becker@ugent.be

'Laboratory of Microbiology, Department of Biochemistry and Microbiology, Faculty of Sciences, Ghent University, Ghent, Belgium

${ }^{2}$ Laboratory of Animal Nutrition, Department of Nutrition, Genetics and Ethology, Faculty of Veterinary Medicine, Ghent University, Merelbeke, Belgium

Full list of author information is available at the end of the article
}

diseases [2-4]. In this context, well-balanced diets represent an important route for prevention or therapeutic intervention $[5,6]$.

Due to diet-induced evolutionary adaptations, cats have developed a strictly carnivorous lifestyle with unique nutrient requirements [7]. Extrapolations of the dietary profile of the domestic cat to wild felids in captivity have been made $[8,9]$ but are highly debatable since great differences exist in regards to their anatomical, behavioral and nutritional characteristics. Domestic cats are subjected to frequent feeding portions of carbohydrate-rich extruded kibble diets [10]. In contrast, captive exotic felids are usually fed once a day a commercially prepared raw meat diet, sometimes supplemented with a vitamin and mineral premix, or whole carcasses [11]. The latter comes with variable

\section{Biomed Central}


amounts of indigestible animal tissues, such as raw bones, tendons, cartilage, skin, hair or feather. The undigested portion of the diet provides the main source of fermentable substrates for the intestinal microbiota, which form the main go-between in the translation of nutritional properties of the diet to health benefits for the host [12]. Comparison of mammalian gut microbiotas has shown that diet is, next to gut physiology, a major regulator of faecal microbiota composition [13].

In domestic cats, taxonomic and functional studies of the intestinal microbial communities have shown that different sources of dietary fibre (i.e., cellulose, pectin, fructooligosaccharide) modified the composition of bacterial phyla in the faeces. For instance, cats fed a diet containing $4 \%$ pectin were found to display a higher percentage of Firmicutes and Spirochaetes than cats fed a diet containing $4 \%$ cellulose [14]. In the same study, dietary fructooligosaccharides increased the percentage of Actinobacteria. Conversely, high-protein diets induced a microbial shift towards decreased E. coli, Bifidobacterium and Lactobacillus populations [15,16]. In captive exotic felids, however, information on the composition and dietary modulation of the intestinal microbiota remains scarce [8].

Recent in vivo and in vitro studies in one of the most endangered exotic felid species, the cheetah (Acinonyx jubatus), point towards a significant role for microbial degradation of undigested animal tissues in the host's metabolic homeostasis $[17,18]$. However, because the number of captive animals available for well-documented faecal sample collection is extremely limited and because the composition and the functional capacity of the cheetah microbiota is virtually unknown, it has not been possible to link these observations to specific bacterial shifts or adaptations in the intestinal ecosystem. In addition, direct extrapolation of microbiological insights obtained for the domestic cat is not a valid approach given its adaptation to commercial diets. To start bridging the knowledge gap between the design of nutritional intervention strategies and the prediction of potential health benefits, this study aimed to inventorize the predominant faecal microbiota of the only two captive cheetahs held in a zoo in Flanders (Belgium) associated with the European Association of Zoos and Aquaria (EAZA). Compositional analysis of $16 \mathrm{~S}$ rRNA gene clone libraries was used for classification of the obtained phylotypes at phylum and family level, leading to the identification of the major bacterial groups that compose the cheetah's intestinal ecosystem.

\section{Methods}

\section{Sample collection}

Fresh faecal samples (200 gram) were collected in 2011 from the two adult male cheetahs (B1 and B2; both 10 years old) housed at Zooparc Planckendael (Flanders,
Belgium), a full member of EAZA (http://www.eaza.net/ membership). The animals shared indoor and outdoor housing and were fed their regular zoo diet i.e. chunked boneless horsemeat ( $2 \mathrm{~kg} /$ day/animal) topdressed with a vitamin and mineral premix (Carnicon; Aveve, Leuven, Belgium) randomly interspersed with unsupplemented whole rabbits. No medical or health problems were reported or apparent on remote examination, and both cheetahs were treated prophylactically for internal parasites (Horseminth; ${ }^{\oplus}$ Pfizer, Brussels, Belgium). Faecal samples were immediately collected upon defaecation into plastic tubes, transported on dry ice and stored at $-80^{\circ} \mathrm{C}$ until further analysis.

\section{DNA extraction}

Prior to DNA extraction, 25 grams (wet weight) of each thawed faecal sample was placed separately in sterile stomacher bags and homogenized in $225 \mathrm{ml}$ peptonebuffered saline (PBS) (0.1\% [wt/vol] bacteriological peptone [L37; Oxoid, Basingstoke, United Kingdom], 0.85\% [wt/vol] NaCl [106404; Merck, Darmstadt, Germany]). The sludgy homogenate was filtered on a Büchner funnel to discard large particles such as hair and bones, and subsequently divided into $1.5 \mathrm{ml}$ aliquots which were stored at $-80^{\circ} \mathrm{C}$.

The protocol of Pitcher et al. [19] was used in a modified version [20] to extract total bacterial DNA from the faecal samples. DNA size and integrity were assessed on 1\% agarose electrophoresis gels stained with ethidium bromide. DNA concentration and purity were determined by spectrophotometric measurement at 234, 260 and $280 \mathrm{~nm}$. DNA extracts were finally diluted ten times with TE buffer (1 mM EDTA [324503; Merck, Darmstadt, Germany], 10 mM Tris-HCl [648317; Merck, Darmstadt, Germany]) and stored at $-20^{\circ} \mathrm{C}$.

\section{Real-time PCR}

Quantitative PCR amplification and detection were performed using the Roche Light Cycler 480 machine with the Roche Light Cycler 480 SYBR Green I Master kit. Each PCR reaction included $40 \mathrm{ng}$ DNA. Specific primers were used for Bacteroidetes (Bact934F [5' GGARCATGTGGTT TAATTCGATGAT 3'] and Bact1060R [5' AGCTGACGA CAACCATGCAG 3']) and Firmicutes (Firm934F [5' GG AGYATGTGGTTTAATTCGAAGCA 3'] and Firm 1060R [5' AGCTGACGACAACCATGCAC 3']), along with universal primers for total bacteria (Eub338F [5' ACTCCTA CGGGAGGCAGCAG 3'] and Eub518R [5' ATTACC GCGGCTGCTGG 3']) as previously described [21]. Samples were incubated at $95^{\circ} \mathrm{C}$ for $5 \mathrm{~min}$ and subsequently amplified during 45 cycles of $95^{\circ} \mathrm{C}$ for $10 \mathrm{~s}, 60^{\circ} \mathrm{C}$ for $30 \mathrm{~s}$, and $72^{\circ} \mathrm{C}$ for $1 \mathrm{~s}$. The relative amount of Firmicutes and Bacteroidetes 16S rRNA in each sample was normalized to the total amount of faecal bacteria amplified with $16 \mathrm{~S}$ 
rRNA gene-based universal primers [22,23]. Bifidobacteriaceae were quantified using Bifidobacterium-specific primers g-Bifid-F (5' CTCCTGGAAACGGGTGG 3') and g-BifidR (5' GGTGTTCTTCCCGATATCTACA 3') [24].

The ability of primers Bact934F and Bact1060R to detect members of the Bacteroidetes phylum in cheetah faeces was evaluated in a spiking experiment. For that purpose, Bacteroides fragilis DSM 1396, Bacteroides uniformis DSM 6597 and Bacteroides distansonius DSM 20701 were cultured anaerobically at $37^{\circ} \mathrm{C}$ for $48 \mathrm{~h}$ on Reinforced Clostridial Medium (RCM) (M37; Oxoid, Basingstoke, United Kingdom). Inocula were prepared from harvested colonies and enumerated by plating serial 10-fold dilutions. Similarly, RCM counts were determined for faecal homogenates of B1 and B2. These homogenates were spiked with an equivalent mixture of the three Bacteroides strains at 1\%, $10 \%$ and $50 \%$ of the total RCM count. Spiked samples were subjected to DNA-extraction and real-time PCR as described above.

\section{Community PCR}

Template DNA obtained from cheetahs B1 and B2 was subjected to $16 \mathrm{~S}$ rRNA gene amplification using the conserved primers pA (5' AGA GTT TGA TCC TGG CTC AG 3') and pH (5' AAG GAG GTG ATC CAG CCG CA 3') which flank respectively the extreme 5' and $3^{\prime}$ part of the $16 \mathrm{~S}$ rRNA gene, thus allowing amplification of the entire gene [25]. Each reaction mixture $(50 \mu \mathrm{l})$ contained $5 \mu \mathrm{l} \mathrm{10x}$ PCR buffer $(100 \mathrm{mM}$ Tris- $\mathrm{HCl}$, $\mathrm{pH} 8.3$ [at $25^{\circ} \mathrm{C}$ ]; $500 \mathrm{mM} \mathrm{KCl} ; 15 \mathrm{mM} \mathrm{MgCl}_{2} ; 0.01 \%$ [wt/ vol] gelatin [GeneAmp; Applied Biosystems, USA]), $1 \mu \mathrm{l}$ $25 \mathrm{mM} \mathrm{MgCl}_{2}$, $5 \mu \mathrm{l} 2 \mathrm{mM}$ dNTPs (GeneAmp; Applied Biosystems, USA), $0.04 \mu \mathrm{l} 10 \mu \mathrm{g} / \mu \mathrm{l}$ bovine serum albumin, $1.25 \mu \mathrm{l} 1 \mathrm{U} / \mu \mathrm{l}$ AmpliTaq ${ }^{\oplus}$ (Applied Biosystems, USA), $2.5 \mu \mathrm{l}$ of each $10 \mu \mathrm{M}$ primer, $4 \mu \mathrm{l}$ template DNA and milliQ water to $50 \mu \mathrm{l}$. The samples were amplified in the Veriti ${ }^{\mathrm{Ta}} \mathrm{Dx}$ 96Well Thermal Cycler (Applied Biosystems, USA), using the following PCR programme: initial denaturation at $94^{\circ} \mathrm{C}$ for $5 \mathrm{~min}$ followed by 18 cycles of $94^{\circ} \mathrm{C}$ for $1 \mathrm{~min}, 55^{\circ} \mathrm{C}$ for $1 \mathrm{~min}$ and $72^{\circ} \mathrm{C}$ for $1 \mathrm{~min}$, with a final extension of $72^{\circ} \mathrm{C}$ for $10 \mathrm{~min}$. Negative (milliQ water as template) and positive controls (Marinobacter sp. strain T278 [R-39409]) were included in parallel. Amplicons were checked on a $1 \%$ agarose gel under UV illumination after ethidium bromide staining of the gel, and subsequently purified with the QIAquick ${ }^{\oplus}$ PCR purification kit (Qiagen, Germany).

\section{Cloning of bacterial 16S rRNA gene amplicons}

For both cheetahs B1 and B2, a clone library was prepared. Purified 16S rRNA gene amplicons were ligated into the $\mathrm{pGEM}^{\bullet}-\mathrm{T}$ Vector System (Promega Benelux, The Netherlands) and transformed into competent $E$. coli cells according to the manufacturer's instructions. White clones were amplified using the primer pair T7
(5' AAT ACG ACT CAC TAT AGG 3') and Sp6 (5' ATT TAG GTG ACA CTA TAG 3') to determine the size of the inserts.

\section{Sequencing and sequence processing}

The diversity of the clone libraries was examined via short fragment sequencing on an ABI PRISM 3130xl Genetic Analyzer (Applied Biosystems, USA) by means of the Big Dye ${ }^{\oplus}$ XTerminator $^{\mathrm{rm}}$ v.3.1. Cycle Sequencing and Purification Kit (Applied Biosystems, USA) according to the protocol of the supplier. The sequencing primer used was BKL1 [26]. For each sample, clones were sequenced, assembled in BioNumerics (Applied Maths, Sint-Martens-Latem, Belgium) and edited to exclude the primer binding sites. Chimeras were detected using Bellerophon [27] and B2C2 [28], and excluded for further analysis.

\section{Phylogenetic analyses}

Chimera-free sequences were aligned using ClustalW in MEGA 5.0 [29] and corrected by manual inspection. Homology searches were performed via BLAST [30], and taxonomic classification of the $16 \mathrm{~S}$ rRNA transcripts was obtained by comparison against The Ribosomal Database Project-II (RDP) [31]. Only annotations with a bootstrap value over 0.8 were considered as well identified phylogenetic levels, leaving successive levels as unclassified. Groups of sequences with $\leq 3 \%$ sequence divergence ( $\geq 97 \%$ similarity) were defined as an operational taxonomic unit (OTU) or phylotype. Rarefaction curves were determined for different clone library sizes and Good's coverage index [32] was calculated as 1-(n/ $\mathrm{N}) \times 100$, where $\mathrm{n}$ is the number of singleton phylotypes and $\mathrm{N}$ is the total number of sequences in the sample. From each OTU at the $97 \%$ cut off, a representative clone was selected along with its nearest type strain from the RDP database. A similarity-matrix was calculated using the Maximum Composite Likelihood parameter and data were visualized in a neighbour-joining phylogenetic tree constructed in MEGA 5.0. Reliability of the tree was evaluated based on 1000 bootstrap replicates.

\section{Availability of supporting data}

The data set supporting the results of this article is available in the GenBank repository, accession numbers KF909375 - KF910074, and the phylogenetic tree has been deposited at TreeBase (http://purl.org/phylo/treebase/phylows/study/TB2:S15139).

\section{Results}

Distribution of OTUs in 16S rRNA gene clone libraries

Two clone libraries (CL-B1 and CL-B2) were created using the full-length 16S rRNA gene amplicons from 
samples B1 and B2. Although most of the DNA inserts corresponded to the expected full-length amplification products, some clones contained short fragments probably due to internal restriction sites. A selection of 384 clones per library was sequenced with primer BKL1, resulting in 352 and 350 quality-checked sequences of 400 to $450 \mathrm{bp}$ length from the $5^{\prime}$ end for libraries CL-B1 and CL-B2, respectively. With a $97 \%$ sequence identity criterion, 29 OTUs were obtained for CL-B1 and 37 OTUs for CL-B2. The coverage of the clone libraries was $98.6 \%$ and $97.7 \%$, respectively, according to Good's formula [32]. Among the 66 OTUs, only 18 were found to be common to both libraries. Together, these common OTUs represented 298 sequences $(84.7 \%)$ in CL-B1 and 317 sequences (90.6\%) in CL-B2. Among the remaining OTUs, 11 OTUs were unique to clone library B1 and 19 to clone library B2. Rarefaction curves were obtained by plotting the number of phylotypes observed from both samples against the number of clones sequenced. The decrease in the rate of phylotype detection indicates that the majority of the predominant bacterial diversity in these samples was covered by clone library analysis [see Additional file 1].

\section{Taxonomic composition of 16S rRNA gene clone libraries at phylum and family level}

Firmicutes was by far the most abundant bacterial phylum representing $96.6 \%$ and $92.9 \%$ of all sequences in CL-B1 and CL-B2, respectively. Three other bacterial phyla formed a minority in the phylogenetic spectrum, i.e. Actinobacteria (3.1\% in CL-B1; $5.4 \%$ in CL-B2), Proteobacteria $(0.3 \%$ in CL-B1; $0.6 \%$ in CL-B2) and Fusobacteria (1.1\% in CL-B2). Surprisingly, none of the sequences was assigned to the Bacteroidetes phylum, a group of gram-negative bacteria that make up a major part of the mammalian distal intestinal microbiota [33]. To validate the results obtained by sequencing, we determined the relative concentrations of Firmicutes and Bacteroidetes with real-time PCR. The Firmicutes/Bacteroidetes ratio for faecal samples of B1 and B2 was 1/ 0.0004 and $1 / 0.0081$, respectively, indicating a very low abundance of Bacteroidetes. In spiked faecal samples, however, Bacteroides spp. were succesfully recovered down to $1 \%\left(10^{4} \mathrm{CFU} / \mathrm{ml}\right)$.

Taxonomic assignment at family level revealed 16 different families of which Clostridiaceae, Ruminococcaceae, Peptococcaceae and the unclassified Clostridiales Incertae Sedis XIV held most representatives. Of all these families, the Clostridiaceae represented by far the highest number of different phylotypes (Figure 1). The distribution of common OTUs within the predominant bacterial families confirms the phylotype richness of Clostridiaceae in both libraries (Table 1).

\section{Phylogenetic analysis of 16S rRNA gene clone libraries at OTU level}

For each OTU, a representative clone sequence was selected along with the type strain of its nearest validated species neighbour as obtained in RDP to construct a wide-range phylogenetic tree. Figure 2 shows the phylogenetic inferences among the OTUs affiliated with the phyla Firmicutes, Actinobacteria, Proteobacteria and Fusobacteria. Recovered sequences within the Firmicutes spanned three major orders i.e. Clostridiales, Lactobacillales and Erysipelotrichales.

Most of the clones fell within the Clostridiales, representing members of seven different bacterial families. A total of 186 clones of this class (31\%) belonged to OTU3 and were highly related ( $<1 \%$ nucleotide divergence) to Clostridium hiranonis TO- $931^{\mathrm{T}}$. Within the Clostridiaceae a high nucleotide similarity was also found for OTU-2, which grouped 65 clones closely to Clostridium perfringens ATCC $13124^{\mathrm{T}}$, and for OTU-34, which clustered with Clostridium fallax ATCC $19400^{\mathrm{T}}$. However, the latter only consisted of one clone and displayed a low bootstrap value of $56 \%$ at its node. For OTU-9, OTU-32 and OTU-5, high bootstrap values (92\%, 100\% and 95\%) and a low nucleotide divergence (1\%) indicated their close phylogenetic affiliation to Clostridium glycyrrhizinilyticum $\mathrm{ZM}^{\mathrm{T}}{ }^{\mathrm{T}}$, Clostridium colicanis DSM $13634^{\mathrm{T}}$ and Clostridium glycolicum DSM $1288^{\mathrm{T}}$, respectively. The remaining five OTUs within the Clostridiaceae family (OTU-31, OTU-1, OTU-30, OTU-33 and OTU-21) clustered under lower bootstrap values with their respective type strains. The Ruminococcaceae family was also well represented by four OTUs of which OTU-7 constituted 89 clones closely related to Ruminococcus gnavus ATCC $29149^{\mathrm{T}}$. The high bootstrap value $(100 \%)$ at the node of cluster OTU-35 and Hydrogenoanaerobacterium saccharovorans SW512 ${ }^{\mathrm{T}}$ suggests a reliable phylogenetic positioning although there was less than $90 \%$ sequence similarity between both. The remaining OTU-19 and OTU-20 included only 6 clones clustering at 5\% nucleotide divergence with Ruminococcus gnavus ATCC $29149^{\mathrm{T}}$ and Ruminococcus torques ATCC $27756^{\mathrm{T}}$, respectively. The Peptococcaceae family was only represented by OTU-6, which included 34 clones and exhibited a low sequence similarity (80\%) with the nearest type strain, Desulfonispora thiosulfatigenes DSM $11270^{\mathrm{T}}$. Moreover, the low bootstrap value (63\%) questions the phylogenetic position of OTU-6 in this tree. The remaining families Lachnospiraceae, Enterococcaceae and Peptostreptococcaceae were represented by 6 different OTUs which together encompassed $6 \%$ of all sequences allocated to the Clostridiales. The unclassified Clostridiales, Incertae Sedis XIV, harbored $18 \%$ of all sequences across three OTUs and were all affiliated to the genus Blautia. However, only OTU-10 showed 1\% sequence divergence to its type strain Blautia hansenii JCM $14655^{\mathrm{T}}$, whereas OTU-12 and OTU-13 differed at least 4\% from the closest 


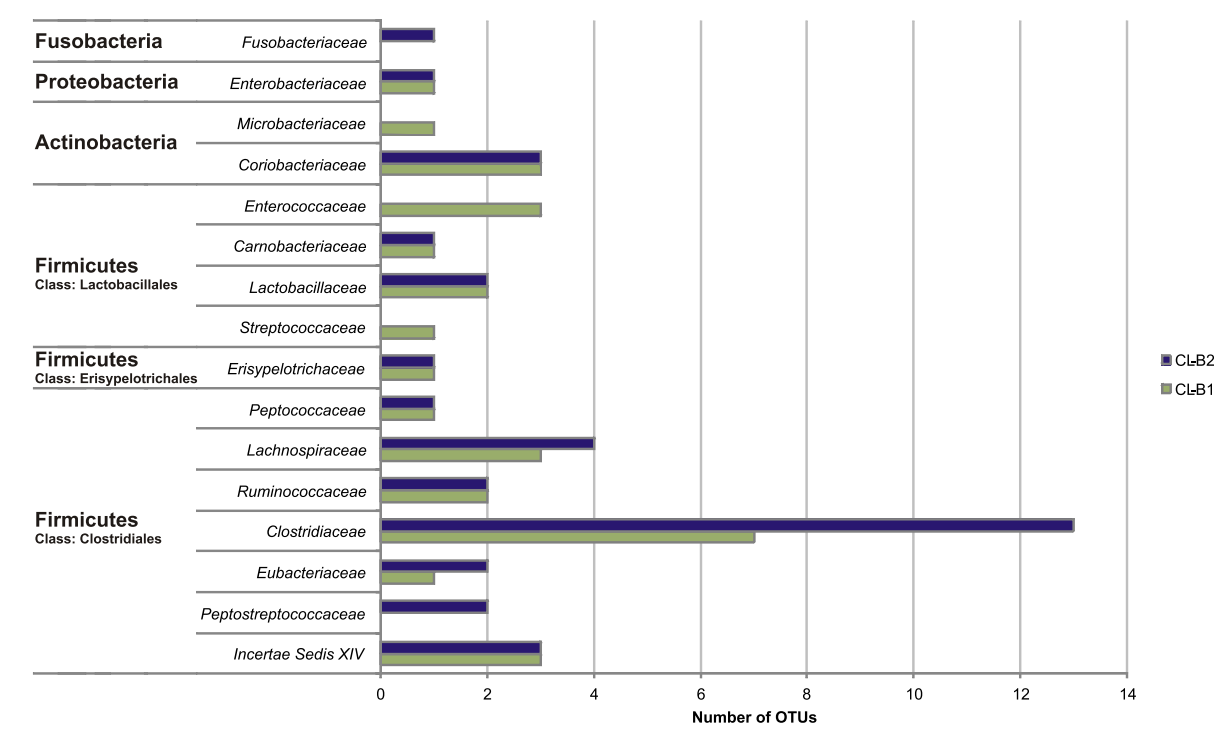

Figure 1 Phylotype frequency at the family level as revealed by clone library analysis of captive cheetah faeces.

relative Blautia glucerasei HFTH-1 ${ }^{\mathrm{T}}$. Based upon the previously proposed classification of Clostridium spp. in phylogenetic clusters [34], Clostridiales sequences from this study fell into three clusters. These included Clostridium cluster XIVa (43\%), which showed the highest OTU variety containing OTU-7 to OTU-13, Clostridium cluster XI (38\%) and Clostridium cluster I (13\%).

Within the Lactobacillales, the bootstrap value of $79 \%$ at the node tenuously supports the grouping in four families. Three OTUs together represented by 36 clones grouped in the Enterococcaceae. Of these, OTU-24 was closely related to Enterococcus hirae DSM $20160^{\mathrm{T}}$ although it only represented one clone with a $3 \%$ nucleotide divergence. The other two OTUs (OTU-23 and OTU-25) differed only 1\% from the sequences of Enterococcus faecalis $\mathrm{JCM} 5803^{\mathrm{T}}$ and Enterococcus cecorum ATCC $43198^{\mathrm{T}}$, respectively. For the Carnobacteriaceae, a monophyletic branch at $100 \%$ bootstrap support was formed by OTU-16 with Carnobacterium divergens DSM $20623^{\mathrm{T}}$. A total of 14 clones all grouping in the Lactobacillaceae formed three subclusters, each at $100 \%$ bootstrap support with their closest type strain. OTU-15 was phylogenetically linked to Lactobacillus sakei DSM $20017^{\mathrm{T}}$, OTU-42 to Lactobacillus mucosae CCUG $43179^{\mathrm{T}}$ and OTU-26 to Lactobacillus animalis NBRC $15882^{\mathrm{T}}$. Finally, Streptococcaceae were represented by OTU-27, which was closely related ( $1 \%$ nucleotide divergence) to Lactococcus piscium CCUG $32732^{\mathrm{T}}$.

The order Erysipelotrichales was divided into two distinct clusters representing members of the Erysipelotrichaceae family. More specifically, OTU-28 (4 clones) grouped most closely to Eubacterium cylindroides ATCC $27803^{\mathrm{T}}$, whereas the single clone of OTU-41 clustered with Turicibacter sanguinis MOL $361^{\mathrm{T}}$.

Table 1 Most abundant OTUs, their taxonomic assignment at family level and closest type strain in number and \% of clones for both clone libraries from captive cheetah faeces

\begin{tabular}{llllll}
\hline OTU $^{\text {a }}$ & Bacterial family & Clostridium cluster & Closest type strain & CL-B1 (352 clones) & CL-B2 (350 clones) \\
\hline OTU-2 & Clostridiaceae & $\mathrm{I}$ & Clostridium perfringens ATCC 13124 $^{\top}$ & $6(1.7 \%)$ & $59(16.9 \%)$ \\
OTU-3 & Clostridiaceae & $\mathrm{XI}$ & Clostridium hiranonis TO-931 $^{\top}$ & $48(13.6 \%)$ & $138(39.4 \%)$ \\
OTU-5 & Clostridiaceae & $\mathrm{XI}$ & Clostridium glycolicum DSM 1288 & $1(0.3 \%)$ & $14(4.0 \%)$ \\
OTU-6 & Peptococcaceae & $\mathrm{n} / \mathrm{a}$ & Desulfonispora thiosulfatigenes DSM 11270 & $33(9.4 \%)$ & $1(0.3 \%)$ \\
OTU-7 & Ruminococcaceae & $\mathrm{XIVa}$ & Ruminococcus gnavus ATCC 29149 & $69(19.6 \%)$ & $20(5.7 \%)$ \\
OTU-10 & Incertae Sedis XIV & $\mathrm{XIVa}$ & Blautia hansenii JCM 14655 & $19(5.4 \%)$ \\
OTU-12 & Incertae Sedis XIV & $\mathrm{XIVa}$ & Blautia glucerasei HFTH-1 & $36(10.2 \%)$ & $3(0.9 \%)$ \\
OTU-13 & Incertae Sedis XIV & XIVa & Blautia glucerasei HFTH-1 & $32(9.1 \%)$ & $8(2.3 \%)$ \\
OTU-17 & Coriobacteriaceae & $\mathrm{n} / \mathrm{a}$ & Collinsella stercoris RCA55-54 & $29(8.2 \%)$ & $13(3.7 \%)$ \\
OTU-25 & Enterococcaceae & $\mathrm{n} / \mathrm{a}$ & Enterococcus cecorum ATCC 43198 & $6(1.7 \%)$ & $31(8.8 \%)$ \\
\hline
\end{tabular}

${ }^{\mathrm{a}} \mathrm{OTU}$ s which consist of at least $\geq 10$ clones in CL-B1 or CL-B2; OTU = operational taxonomic unit; $\mathrm{n} / \mathrm{a}=$ not applicable. 


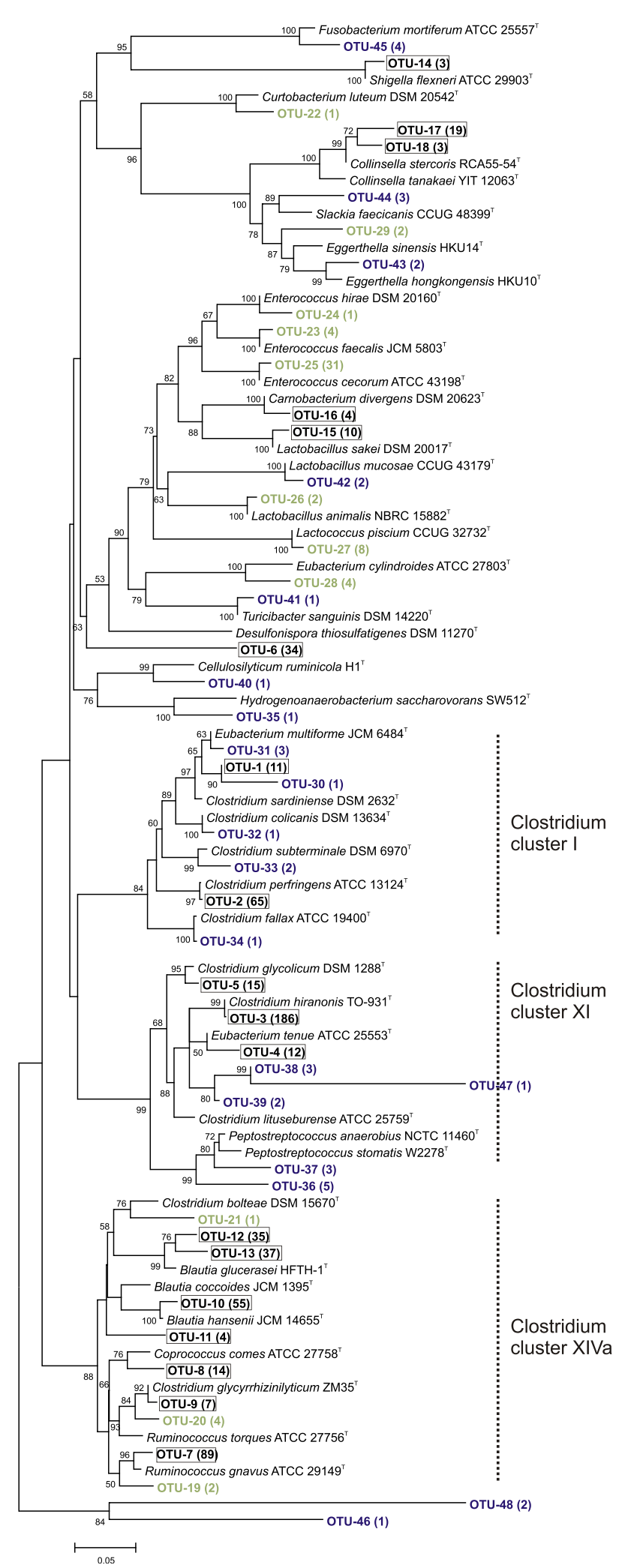

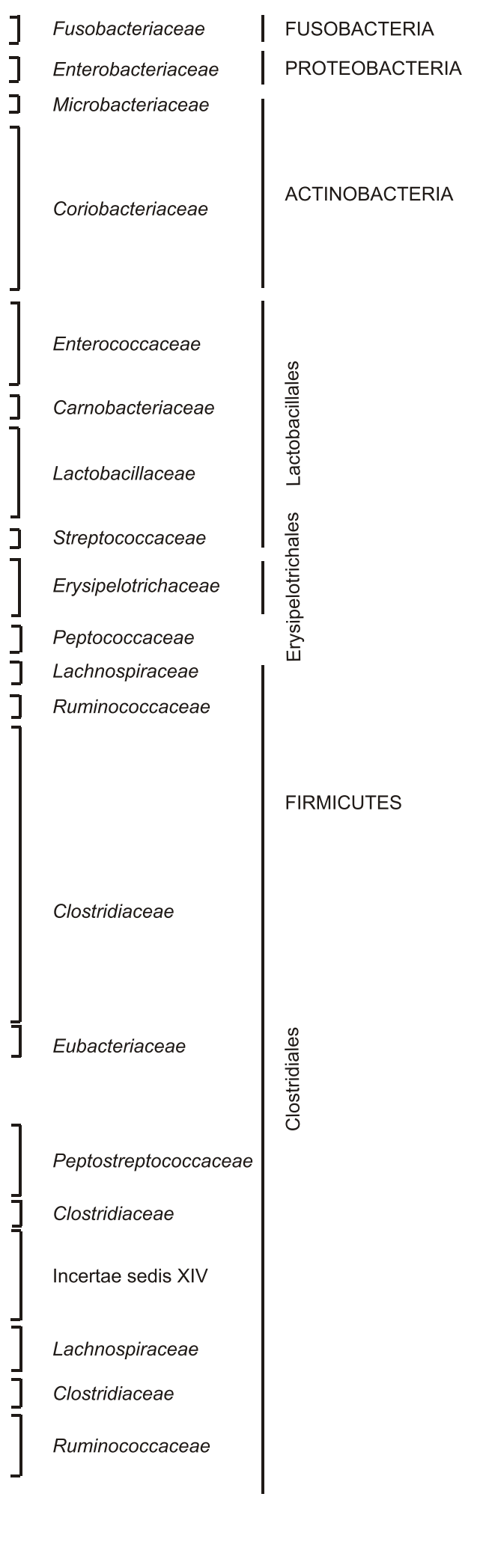

Figure 2 (See legend on next page.) 
(See figure on previous page.)

Figure 2 Neighbour-joining phylogenetic tree showing the nearest phylogenetic related type strains for recovered OTUs from two $16 \mathrm{~S}$ rRNA clone libraries from captive cheetah faeces. Bootstrap values, expressed as percentages of 1000 replications, above $50 \%$ are given at branching points. The scale bar shows 5 nucleotide substitutions per 100 nucleotides. Number of clones in parentheses follows label of either common OTUs (framed), OTUs solely from CL-B1 (green) or CL-B2 (purple).

The branching pattern within the phylum Actinobacteria consisted of two families. The Microbacteriaceae were represented by a single clone (OTU-22) clustering at $100 \%$ bootstrap support with Curtobacterium luteum DSM $20542^{\mathrm{T}}$. The Coriobacteriaceae comprising the genera Collinsella, Slackia and Eggerthella were represented by five OTUs. Of these, OTU-17 (19 clones) and OTU-18 (3 clones) clustered with Collinsella stercoris RCA55-54 ${ }^{\mathrm{T}}$ and Collinsella tanakaei YIT $12063^{\mathrm{T}}$, respectively. The few clones assigned to OTU-29, OTU-43 and OTU-44 were most closely related to Eggerthella hongkongenis $\mathrm{HKU}_{10}^{\mathrm{T}}$, Eggerthella sinensis $\mathrm{HKU} 14^{\mathrm{T}}$ and Slackia faecicanis CCUG $48399^{\mathrm{T}}$, respectively.

The single OTU belonging to the Proteobacteria, OTU-14 (3 clones), exhibited $<2 \%$ nucleotide divergence with Shigella flexneri ATCC $29903^{\mathrm{T}}$ with $100 \%$ bootstrap support. Likewise, the phylum Fusobacteria was only represented by OTU-45 (4 clones), which was phylogenetically most closely related to Fusobacterium mortiferum ATCC $25557^{\mathrm{T}}$.

Five OTUs (OTU-38, OTU-39, OTU-46, OTU-47, OTU-48), containing 1 to 3 clones each, failed to clearly group within a particular genus or family. Given that all sequences used for phylogenetic analyses were of good quality, these OTUs may represent species that are currently not included in the RDP database.

\section{Common diversity of CL-B1 and CL-B2}

The faecal community members shared by CL-B1 and CL-B2 encompassed three phyla (Firmicutes, Actinobacteria and Proteobacteria), 10 families and 18 OTUs (OTU-1 to OTU-18). The Clostridiaceae family harbored five common OTUs. Of these, OTU-3 (affiliated with Clostridium hiranonis $\mathrm{TO}-931^{\mathrm{T}}$ ) accounted for $13.6 \%$ and $39.4 \%$ of all clones in CL-B1 and CL-B2, respectively. Followed by OTU-7 (affiliated with Ruminococcus gnavus ATCC $29149^{\mathrm{T}}$ ) representing $19.6 \%$ and $5.7 \%$ of all sequences in CL-B1 and CL-B2, respectively (Table 1). On top of the five common OTUs, CL-B2 harbored eight unique OTUs within the family Clostridiaceae compared to one unique OTU (OTU-21) for CL-B1. Other shared families within the phylum Firmicutes were the Peptococcaceae, Eubacteriaceae, Lachnospiraceae and unclassified Clostridiales. All of these consisted of common OTUs with the exception of the Lachnospiraceae family that also comprised a single clone of OTU-40 in CL-B2. However, the phylogenetic position of OTU-40 displayed $8 \%$ nucleotide divergence with the closest type strain, Cellulosilyticum ruminicola $\mathrm{H} 1^{\mathrm{T}}$. In the Proteobacteria, only the family Enterobacteriaceae was represented with a single common OTU-14 (affiliated with Shigella flexneri ATCC $29903^{\mathrm{T}}$ ), which harbored a minority population of three clones. The phylum Actinobacteria was represented by two common OTUs (OTU-17 and OTU-18) that were phylogenetically related to the Coriobacteriaceae.

\section{Comparison with available 16S rRNA sequences from captive cheetahs}

Our dataset of 702 quality-checked sequences was compared with 597 full-length 16S RNA gene sequences retrieved from a large comparative microbiome study of Ley and co-workers [35] in which one faecal sample each of two captive cheetahs from Saint Louis Zoo (St Louis, Missouri, USA) were included. Despite differences in sequence number and sequence length, both datasets were compared with taxonomic RDP annotation. In line with the present study, Bacteroidetes represented only a very marginal share (i.e. 1.3\%) in Ley et al.'s dataset. At family level, the dominance of Clostridiaceae (16.5\%) and Ruminococcaceae (4.0\%) members was also confirmed. The share of Peptococcaceae (1.7\%) and the unclassified Clostridiales Incertae Sedis $(0.8 \%)$ in Ley et al.'s dataset was considerably lower compared to our dataset (5\% and $18 \%$, respectively). Two other bacterial families, also represented in the dataset of this study, made up a big part of Ley et al.'s dataset, Peptostreptococcaceae (13\%) and Lachnospiraceae (11\%).

Taken together, only the Clostridiaceae, Lactobacillaceae and Erysipelotrichaceae families were common to the faecal microbiota of all four cheetahs included in these two studies.

\section{Discussion}

This study set out to determine the predominant faecal microbial communities of captive cheetahs using $16 \mathrm{~S}$ rRNA gene clone libraries. At the onset of the study, only two animals with well-documented dietary and health records and housed according to EAZA standards were available for this study in Flanders, Belgium. Phylogenetic analysis of the pooled library set revealed a highly complex microbiota covering a broad phylogenetic spectrum. The Firmicutes were by far the most abundant bacterial phylum compared to the minority of Actinobacteria, Fusobacteria and Proteobacteria. Surprisingly, none of the OTUs of both clone libraries were 
assigned to members of the Bacteroidetes, the phylum that together with the Firmicutes accounts for $>98 \%$ of the $16 \mathrm{~S}$ rRNA gene sequences detected in the gut microbiota of vertebrates [13]. The Bacteroidetes comprise important degraders of complex and otherwise indigestible dietary polysaccharides in the large intestine, which leads to the production of short-chain fatty acids that are reabsorbed by the host as energy source [36,37]. Using a variety of methods, Bacteroidetes have been identified as a dominant group in the faecal microbiota of dogs (27-34\%) fed experimental diets (30\% protein and $20 \%$ fat) $[38,39]$, wild wolves $(16,9 \%)$ feeding on raw meat [40] and grizzly bears (40\%) on an omnivorous diet [41]. Feline microbiome studies using $16 \mathrm{~S}$ rRNA clone libraries or pyrosequencing have also reported that Bacteroidetes is one of the major $(0.45 \%-10 \%)$ phyla in the faecal microbiota of cats alongside Firmicutes and Actinobacteria [42,43]. A recent study using 454 pyrosequencing even reported Bacteroidetes to be the most predominant $(68 \%)$ bacterial phylum in the feline intestinal microbiome [44]. Although relative levels of the dominant phyla in cats seem to vary between studies, likely as a result of differences in methodologies and/or in dietary regimes of the studied cats, one could expect to also find Bacteroidetes in most other felids. The complete absence of Bacteroidetes members in the $16 \mathrm{~S}$ rRNA clone libraries of the two captive cheetahs contradicts this expectation, but was corroborated by real-time PCR data indicating a hardly detectable concentration of this phylum against a high background of Firmicutes. The finding that Bacteroides spp. could be detected in spiked faecal samples at $10^{4} \mathrm{CFU} / \mathrm{ml}$ and possibly lower, excludes major detection artefacts introduced during DNA extraction. Further support for our observations are provided by a comparative study of the gut-associated bacterial communities in 60 mammalian species showing that Bacteroidetes is a rare phylum in most carnivores [35]. In that study, 3-15\% of the $16 \mathrm{~S}$ rRNA gene sequences of captive lions, hyenas and bush dogs were phylogenetically linked to Bacteroidetes, whereas only a marginal contribution $(<1 \%)$ of this phylum was found for captive polar bears and cheetahs. This is comparable to Bacteroidetes levels reported in a recent microbiome study of captive polar bears [45] and our findings for captive cheetahs. The common denominator between the latter two strict carnivores is their protein-rich diet, whereas domestic cats are usually fed commercially prepared diets containing moderate quantities of carbohydrates and plant-derived soluble fibres [46]. This seems to suggest that differences in dietary regimes and feeding habits account for the large variation in Bacteroidetes levels among carnivores. Low proportions of Bacteroidetes have also been reported in giant pandas which belong to the order Carnivora and have a simple intestinal tract, but are feeding on bamboo [47]. Despite their herbivorous lifestyle, studies have shown that the panda faecal microbiota is more similar to other Carnivora than to unrelated herbivores suggesting that next to diet also gut physiology is a regulator of the faecal microbiota composition [13,35].

Within the Firmicutes, the majority of the Clostridiales isolates common to both clone libraries was assigned to Clostridium clusters XIVa (43\%), XI (38\%) and I (13\%). Our results are consistent with previous studies that reported a high prevalence of these three Clostridium clusters in carnivores [48,49]. Likewise, similar distributions were found in feline microbiome studies using $16 \mathrm{~S}$ rRNA clone libraries $[43,50]$ or $16 \mathrm{~S}$ rRNA gene pyrosequencing [42]. Also in the two cheetahs studied by Ley and co-workers [35], similar high abundances of Clostridium clusters XIVa and XI were found in two other cheetahs. Clostridium cluster XIVa constitutes a major and highly diverse bacterial group in the distal intestines of mammals [51]. This phylogenetically heterogeneous cluster is in both clone libraries represented by Ruminococcaceae spp. most closely related to known mucindegrading organisms such as Ruminococcus torques and Ruminococcus gnavus [52] as well as members of the recently proposed genus Blautia [53]. The latter group comprises important producers of short-chain fatty acids such as butyrate, which is an important source of energy for colonic epithelial cells and has shown to possess antiinflammatory and anticarcinogenic potential $[54,55]$. Feline and canine inflammatory bowel diseases have been associated with reduced bacterial species richness and a reduced proportion of Clostridium cluster XIVa [56-58]. Noteworthy, the two cheetahs included in our study showed no signs of gastrointestinal disease. Clostridium clusters XI and I include saccharolytic fibre-fermenting species but also proteolytic or toxinogenic clostridia [34]. In Clostridium cluster XI, 87\% of the common sequences displayed $>99 \%$ sequence similarity to the type strain of Clostridium hiranonis. This species was first described in human faeces and displays bile acid 7- $\alpha$-dehydroxylating activity. In addition, acetic acid and minor amounts of propionic acid and iso-butyric acid are produced from mono- and disaccharides [59]. Ritchie and co-workers [43] found Clostridium cluster XI to account for $22 \%$ of the faecal microbiota in healthy cats. Up to $86 \%$ of the clones assigned to Clostridium cluster I in our study were phylogenetically most closely related to the type strain of the potentially pathogenic species Clostridium perfringens. However, with reported isolation rates of up to $63 \%$ in healthy cats [60], C. perfringens should probably be considered as a common commensal of the feline intestine. Moreover, no significant differences in prevalence of either C. perfringens or toxigenic C. perfringens strains were observed between healthy cats and cats with diarrhea [60]. 
Protein-rich diets may increase the presence of Clostridium cluster I in pet cats and dogs and induce a shift towards a higher prevalence of proteolytic bacterial species $[16,61]$. A similar dietary influence has also been reported in other carnivores. Clostridium cluster I and XI prevailed in polar bears feeding on seals and fish [45] and captive grizzly bears feeding on a regular diet containing up to $31 \%$ protein [49]. The latter study indicated that captive grizzly bears consuming a protein-based diet were more prone to carry $C$. perfringens than wild grizzly bears consuming a more plant-based diet. These results suggest a positive correlation between the prevalence of Clostridium clusters I and XI and dietary protein content. In the present study, both cheetahs included in our study were fed a protein-rich diet with minimal dietary fibre i.e. boneless horsemeat. Therefore, the high proportions of Clostridium cluster I and XI in the faecal microbiota of captive cheetahs may be a reflection of their dietary habits.

Common bacterial communities classified in the phylum Actinobacteria harbored solely species belonging to the genus Collinsella within the Coriobacteriaceae. This family is a frequent resident of the feline gut microbiota [62]. No members were identified of the Bifidobacteriaceae, a group of fibre-fermenting gut bacteria that largely contribute to cross-feeding mechanisms leading to the production of butyrate $[63,64]$. Also in two other studies both using $16 \mathrm{~S}$ rRNA gene clone libraries to study the faecal microbiota of wild wolves [40] and pet cats [50], no Bifidobacteriaceae were encountered. In contrast, other studies have reported the presence of Bifidobacteriaceae in the feline faecal microbiota using alternative techniques such as culturing [65], FISH [56] and a chaperonin 60 gene-based clone library [66]. This suggests that differences in methodologies may, at least to some extent, explain the observed differences between studies. In fact, it has been shown that Bifidobacteriaceae may be underrepresented in $16 \mathrm{~S}$ rRNA gene-based studies, possibly due to the use of universal primers that may underestimate the GC-rich Actinobacteria. Therefore, the combined use of universal and genus-specific primers has been suggested to characterize Bifidobacterium spp. in intestinal microbiota $[43,67,68]$. In the present study, real-time PCR enumeration of Bifidobacterium revealed a low mean $\log _{10}$ number of 4.43 (data not shown). On the one hand, this illustrates the inability of the clone library approach to detect low levels of Bifidobacterium in the cheetah faecal samples. On the other hand, the finding of a significantly higher mean $\log _{10}$ Bifidobacterium concentration of 9.13 in faecal samples of five domestic cats with the same real-time PCR protocol (Becker et al., unpublished data) indicates that marked differences exist in bifidobacterial levels of cheetahs and domestic cats. Possibly, these differences reflect the strictly carnivorous diet of captive cheetahs. In fact, Bifidobacteriaceae have been negatively correlated with the protein content of the diet $[16,69]$ and only a few studies have reported the presence of bifidobacteria in faeces of carnivores [70].

Finally, the minor share of Fusobacteria and Proteobacteria found in this study is also confirmed in other feline microbiome studies using $16 \mathrm{~S}$ rRNA gene clone libraries [50] or shotgun sequencing [44]. Felids seem to harbor less Proteobacteria and Fusobacteria compared to other carnivores such as wolves [40] and dogs. In the latter species even, substantial numbers of Fusobacteria have been observed, but the significance of an enriched Fusobacteria population is yet unknown [39]. In the Proteobacteria, a minority of three clones affiliated with Shigella flexneri ATCC $29903^{\mathrm{T}}$. This species is principally a primate pathogen causing bacillary dysentery or shigellosis [71]. Cats have not been reported to be naturally infected [72], although these organisms may be transiently excreted in some clinically normal domestic cats $[43,44]$. The two cheetahs included in this study showed no signs of shigellosis and to our knowledge this type of infection has not been reported in cheetahs thus far.

\section{Conclusions}

This is the first ever study to specifically characterize the predominant faecal bacterial populations of captive cheetahs using a combination of 16S rRNA clone library and real-time PCR analyses. The study revealed a complex microbial diversity predominantly composed of Firmicutes. The abundance of Clostridium clusters XIVa, $\mathrm{XI}$ and I in this phylum resembles that in the faecal microbiota of other carnivores. However, the near absence of Bacteroidetes and the low abundance of Bifidobacteriaceae are in sharp contrast with the situation in domestic cats but in agreement with faecal microbiota composition reported in other Carnivora. In addition to the apparent differences in feeding habbits between both felid species, also our microbiological findings thus question the role of the domestic cat as a suitable model for nutritional intervention studies in captive felids such as cheetahs.

The present study provides a first taxonomic baseline for further characterizations of the diversity and dynamics of the cheetah intestinal ecosystem. To confirm our main findings based on two animals, the collection of fresh and well-documented faecal samples from more captive cheetahs worldwide is the next challenge. Ultimately, the resulting microbial insights may contribute in the optimization of feeding strategies and the improvement of the general health status of cheetahs in captivity.

\section{Additional file}

Additional file 1: Rarefaction curves for bacterial 16S rRNA gene sequences obtained by clone library analysis of captive cheetah faecal samples. The slopes of corresponding lineair lines indicate a 
flattening of the rarefaction curves. CL-B1: clone library of faecal samples of captive cheetah B1; CL-B2: clone library of faecal samples of captive cheetah B2.

\section{Competing interests}

The authors declare no conflict of interest.

\section{Authors' contributions}

GH, GPJJ and MH designed and supervised the study. AAMJB performed sample collection; AAMJB and JH performed clone library and sequence analysis; AAMJB and GH were responsible for the draft and final version of the manuscript. All authors read and approved the final manuscript.

\section{Acknowledgements}

Our work was kindly supported by the Special Research Fund of Ghent University (Belgium) and by the Morris Animal Foundation (Grant D12ZO404). The authors gratefully acknowledge zookeepers and staff from Zooparc Planckendael (Flanders, Belgium) for their cooperation during this study. In particular, we thank the cheetah keepers for being sympathetic to this research and for their assistance during the sampling. A special thanks goes out to Arne Vandewalle for his assistance during sample collection. We also wish to thank Dr. Sarah Depauw for her advice and expertise on faecal sampling and Dr. Brigitta Brinkman for her advice and assistance during real-time PCR analyses.

\section{Author details}

'Laboratory of Microbiology, Department of Biochemistry and Microbiology, Faculty of Sciences, Ghent University, Ghent, Belgium. '2aboratory of Animal Nutrition, Department of Nutrition, Genetics and Ethology, Faculty of Veterinary Medicine, Ghent University, Merelbeke, Belgium. ${ }^{3}$ BCCM/LMG Bacteria Collection, Faculty of Sciences, Ghent University, Ghent, Belgium.

Received: 7 May 2013 Accepted: 24 January 2014

Published: 18 February 2014

\section{References}

1. Kawata K: Zoo animal feeding: a natural history viewpoint. Der Zool Garten 2008, 78:17-42.

2. Munson L, Terio K, Worley M, Jago M, Bagot-Smith A, Marker L: Extrinsic factors significantly affect patterns of disease in free-ranging and captive cheetah (Acinonyx jubatus) populations. J Wildl Dis 2005, 41:542-548.

3. Allen ME, Ullrey DE: Relationships among nutrition and reproduction and relevance for wild animals. Zoo Biol 2004, 23:475-487.

4. Kotsch V, Kubber-Heiss A, Url A, Walzer C, Schmidt R: Diseases of captive cheetahs (Acinonyx jubatus) within the European Endangered Species Program (EEP) - a 22-year retrospective histopathological study. Wien Tierarztl Monatsschr 2002, 89:341-350.

5. Garcia-Mazcorro JF, Lanerie DJ, Dowd SE, Paddock CG, Grutzner N, Steiner $J M$, Ivanek R, Suchodolski JS: Effect of a multi-species synbiotic formulation on fecal bacterial microbiota of healthy cats and dogs as evaluated by pyrosequencing. FEMS Microbiol Ecol 2011, 78:542-554.

6. Gaggia F, Mattarelli P, Biavati B: Probiotics and prebiotics in animal feeding for safe food production. Int J Food Microbiol 2010, 141:S15-S28.

7. Morris JG: Idiosyncratic nutrient requirements of cats appear to be diet-induced evolutionary adaptations. Nutr Res Rev 2002, 15:153-168.

8. Vester BM, Beloshapka AN, Middelbos IS, Burke SL, Dikeman CL, Simmons LG, Swanson KS: Evaluation of nutrient digestibility and fecal characteristics of exotic felids fed horse- or beef-based diets: use of the domestic cat as a model for exotic felids. Zoo Biol 2010, 29:432-448.

9. Dierenfeld ES: Nutrition of captive cheetahs - food composition and blood parameters. Zoo Biol 1993, 12:143-150.

10. Zoran DL, Buffington CAT: Effects of nutrition choices and lifestyle changes on the well-being of cats, a carnivore that has moved indoors. J Am Vet Med Assoc 2011, 239:596-606.

11. Vester BM, Swanson KS, Fahey GC: Nutrition of the Exotic Felid. Feedstuffs 2009 (20):57-59.

12. Hooper LV, Midtvedt T, Gordon Jl: How host-microbial interactions shape the nutrient environment of the mammalian intestine. Annu Rev Nutr 2002, 22:283-307.
13. Ley RE, Lozupone CA, Hamady M, Knight R, Gordon Jl: Worlds within worlds: evolution of the vertebrate gut microbiota. Nat Rev Microbiol 2008, 6:776-788.

14. Barry KA, Wojcicki BJ, Middelbos IS, Vester BM, Swanson KS, Fahey GC: Dietary cellulose, fructooligosaccharides, and pectin modify fecal protein catabolites and microbial populations in adult cats. J Anim Sci 2010, 88:2978-2987.

15. Vester BM, Dalsing BL, Middelbos IS, Apanavicius CJ, Lubbs DC, Swanson KS: Faecal microbial populations of growing kittens fed high- or moderate-protein diets. Arch Anim Nutr 2009, 63:254-265.

16. Lubbs DC, Vester BM, Fastinger ND, Swanson KS: Dietary protein concentration affects intestinal microbiota of adult cats: a study using DGGE and qPCR to evaluate differences in microbial populations in the feline gastrointestinal tract. J Anim Physiol Anim Nutr (Berl) 2009, 93:113-121.

17. Depauw S, Bosch G, Hesta M, Whitehouse-Tedd K, Hendriks WH, Kaandorp J, Janssens GPJ: Fermentation of animal components in strict carnivores: a comparative study with cheetah fecal inoculum. J Anim Sci 2012, 90:2540-2548

18. Depauw S, Hesta M, Whitehouse-Tedd K, Vanhaecke L, Verbrugghe A, Janssens GPJ: Animal fibre: The forgotten nutrient in strict carnivores? First insights in the cheetah. J Anim Physiol Anim Nutr (Berl) 2013, 97:146-154

19. Pitcher DG, Saunders N, Owen RJ: Rapid extraction of bacterial genomic DNA with guanidium thiocyanate. Lett Appl Microbiol 1989, 8:151-156.

20. Vanhoutte T, Huys G, De Brandt E, Swings J: Temporal stability analysis of the microbiota in human feces by denaturing gradient gel electrophoresis using universal and group-specific 16S rRNA gene primers. FEMS Microbiol Ecol 2004, 48:437-446.

21. Brinkman BM, Hildebrand F, Kubica M, Goosens D, Del Favero J, Declercq W, Raes J, Vandenabeele P: Caspase deficiency alters the murine gut microbiome. Cell Death Dis 2011, 2:e220.

22. Fierer $N$, Jackson JA, Vilgalys $R$, Jackson RB: Assessment of soil microbial community structure by use of taxon-specific quantitative PCR assays. Appl Environ Microbiol 2005, 71:4117-4120.

23. Guo X, Xia X, Tang R, Zhou J, Zhao H, Wang K: Development of a real-time PCR method for Firmicutes and Bacteroidetes in faeces and its application to quantify intestinal population of obese and lean pigs. Lett Appl Microbiol 2008, 47:367-373.

24. Matsuki T, Watanabe K, Fujimoto J, Kado Y, Takada T, Matsumoto K, Tanaka R: Quantitative PCR with $16 \mathrm{~S}$ rRNA-gene-targeted species-specific primers for analysis of human intestinal bifidobacteria. Appl Environ Microbiol 2004, 70:167-173.

25. Edwards U, Rogall T, Blöcker H, Emde M, Böttger EC: Isolation and direct complete nucleotide determination of entire genes. Characterization of a gene conding for 165 ribosomal RNA. Nucleic Acids Res 1989, 17:7843-7853.

26. Coenye T, Falsen E, Vancanneyt M, Hoste B, Govan JR, Kersters K Vandamme P: Classification of Alcaligenes faecalis-like isolates from the environment and human clinical samples as Ralstonia gilardii sp. nov. Int J Syst Bacteriol 1999, 49(2):405-413.

27. Huber T, Faulkner G, Hugenholtz P: Bellerophon: a program to detect chimeric sequences in multiple sequence alignments. Bioinformatics 2004, 20:2317-2319

28. Gontcharova V, Youn E, Wolcott RD, Hollister EB, Gentry TJ, Dowd SE: Black box chimera check (B2C2): a windows-based software for batch depletion of chimeras from bacterial 16S datasets. Open Microbiol J 2010, 4:47-52.

29. Tamura K, Peterson D, Peterson N, Stecher G, Nei M, Kumar S: MEGA5: Molecular Evolutionary Genetics Analysis Using Maximum Likelihood, Evolutionary Distance, and Maximum Parsimony Methods. Mol Biol Evol 2011, 28:2731-2739.

30. Dorman N: Citations. Biotechniques 2012, 52:403-410.

31. Cole JR, Wang Q, Cardenas E, Fish J, Chai B, Farris RJ, Kulam-Syed-Mohideen AS, McGarrell DM, Marsh T, Garrity GM, Tiedje JM: The ribosomal database project: improved alignments and new tools for rRNA analysis. Nucleic Acids Res 2009, 37(Database issue):D141-145.

32. Good IJ: The population frequencies of species and the estimation of population parameters. Biometrika 1953, 40:237-264.

33. Sekirov I, Russell SL, Antunes LCM, Finlay BB: Gut microbiota in health and disease. Physiol Rev 2010, 90:859-904.

34. Collins MD, Lawson PA, Willems A, Cordoba JJ, Fernandezgarayzabal J Garcia P, Cai J, Hippe H, Farrow JAE: The phylogeny of the genus Clostridium proposal of 5 new genera and 11 new species combinations. Int J Syst Bacteriol 1994, 44:812-826. 
35. Ley RE, Hamady M, Lozupone C, Turnbaugh PJ, Ramey RR, Bircher JS, Schlegel ML, Tucker TA, Schrenzel MD, Knight R, Gordon Jl: Evolution of mammals and their gut microbes. Science 2008, 320:1647-1651.

36. Thomas F, Hehemann J-H, Rebuffet E, Czjzek M, Michel G: Environmental and gut bacteroidetes: the food connection. Front Microbiol 2011, 2:93.

37. Tremaroli V, Bäckhed F: Functional interactions between the gut microbiota and host metabolism. Nature 2012, 489:242-249.

38. Middelbos IS, Vester Boler BM, Qu A, White B, Swanson KS, Fahey GC: Phylogenetic characterization of fecal microbial communities of dogs fed diets with or without supplemental dietary fiber using 454 pyrosequencing. PLoS One 2010, 5:e9768.

39. Swanson KS, Dowd SE, Suchodolski JS, Middelbos IS, Vester BM, Barry KA Nelson KE, Torralba M, Henrissat B, Coutinho PM, Cann IKO, White BA, Fahey GC: Phylogenetic and gene-centric metagenomics of the canine intestinal microbiome reveals similarities with humans and mice. ISME J 2011, 5:639-649.

40. Zhang HH, Chen L: Phylogenetic analysis of $16 \mathrm{~S}$ rRNA gene sequences reveals distal gut bacterial diversity in wild wolves (Canis lupus). Mol Biol Rep 2010, 37:4013-4022.

41. Schwab C, Cristescu B, Boyce MS, Stenhouse GB, Ganzle M: Bacterial populations and metabolites in the feces of free roaming and captive grizzly bears. Can J Microbiol 2009, 55:1335-1346.

42. Handl S, Dowd SE, Garcia-Mazcorro JF, Steiner JM, Suchodolski JS: Massive parallel 16S rRNA gene pyrosequencing reveals highly diverse fecal bacterial and fungal communities in healthy dogs and cats. FEMS Microbiol Ecol 2011, 76:301-310

43. Ritchie LE, Burke KF, Garcia-Mazcorro JF, Steiner JM, Suchodolski JS: Characterization of fecal microbiota in cats using universal 16S rRNA gene and group-specific primers for Lactobacillus and Bifidobacterium spp. Vet Microbiol 2010, 144:140-146.

44. Tun HM, Brar MS, Khin N, Jun L, Hui RKH, Dowd SE, Leung FCC: Genecentric metagenomics analysis of feline intestinal microbiome using 454 junior pyrosequencing. J Microbiol Methods 2012, 88:369-376.

45. Schwab C, Gänzle M: Comparative analysis of fecal microbiota and intestinal microbial metabolic activity in captive polar bears. Can J Microbiol 2011, 57:177-185.

46. Zoran DL: The carnivore connection to nutrition in cats. J Am Vet Med Assoc 2002, 221:1559-1567.

47. Wei G, Lu H, Zhou Z, Xie H, Wang A, Nelson K, Zhao L: The microbial community in the feces of the giant panda (Ailuropoda melanoleuca) as determined by PCR-TGGE profiling and clone library analysis. Microb Ecol 2007, 54:194-202.

48. Suchodolski JS, Camacho J, Steiner JM: Analysis of bacterial diversity in the canine duodenum, jejunum, ileum, and colon by comparative $16 \mathrm{~S}$ rRNA gene analysis. FEMS Microbiol Ecol 2008, 66:567-578.

49. Schwab C, Cristescu B, Northrup JM, Stenhouse GB, Gänzle M: Diet and environment shape fecal bacterial microbiota composition and enteric pathogen load of grizzly bears. PLoS One 2011, 6:e27905.

50. Ritchie LE, Steiner JM, Suchodolski JS: Assessment of microbial diversity along the feline intestinal tract using 16S rRNA gene analysis. FEMS Microbiol Ecol 2008, 66:590-598.

51. Hayashi H, Sakamoto M, Kitahara M, Benno Y: Diversity of the Clostridium coccoides group in human fecal microbiota as determined by 16S rRNA gene library. FEMS Microbiol Lett 2006, 257:202-207.

52. Hoskins LC: Mucin degradation in the human gastrointestinal tract and its significance to enteric microbial ecology. Eur J Gastroenterol Hepatol 1992, 5:205-213.

53. Liu C, Finegold SM, Song Y, Lawson P: Reclassification of Clostridium coccoides, Ruminococcus hansenii, Ruminococcus hydrogenotrophicus, Ruminococcus luti, Ruminococcus productus and Ruminococcus schinkii as Blautia coccoides gen. nov., comb. nov., Blautia hansenii comb. nov., Blautia hydroge. Int J Syst Evol Microbiol 2008, 58(Pt 8):1896-1902.

54. Barcenilla A, Pryde SE, Martin JC, Duncan H, Stewart CS, Henderson C, Harry J, Duncan SH, Flint HJ: Phylogenetic relationships of butyrate-producing bacteria from the human gut. Appl Environ Microbiol 2000, 66:1654-1661.

55. Meijer K, De Vos P, Priebe MG: Butyrate and other short-chain fatty acids as modulators of immunity: what relevance for health? Curr Opin Clin Nutr Metab Care 2010, 13:715-721.

56. Inness VL, McCartney AL, Khoo C, Gross KL, Gibson GR: Molecular characterisation of the gut microflora of healthy and inflammatory bowel disease cats using fluorescence in situ hybridisation with special reference to Desulfovibrio spp. J Anim Physiol Anim Nutr (Berl) 2007, 91:48-53.

57. Janeczko S, Atwater D, Bogel E, Greiter-Wilke A, Gerold A, Baumgart M, Bender H, McDonough PL, McDonough SP, Goldstein RE, Simpson KW: The relationship of mucosal bacteria to duodenal histopathology, cytokine mRNA, and clinical disease activity in cats with inflammatory bowel disease. Vet Microbio/ 2008, 128:178-193.

58. Suchodolski JS, Dowd SE, Wilke V, Steiner JM, Jergens AE: 16S rRNA gene pyrosequencing reveals bacterial dysbiosis in the duodenum of dogs with idiopathic inflammatory bowel disease. PLoS One 2012, 7:e39333.

59. Kitahara M, Takamine F, Imamura T, Benno Y: Clostridium hiranonis sp. nov., a human intestinal bacterium with bile acid 7alpha-dehydroxylating activity. Int J Syst Evol Microbiol 2001, 51(1):39-44.

60. Queen EV, Marks SL, Farver TB: Prevalence of selected bacterial and parasitic agents in feces from diarrheic and healthy control cats from Northern California. J Vet Intern Med 2012, 26:54-60.

61. Zentek J, Fricke S, Hewicker-trautwein M, Ehinger B, Amtsberg G, Baums C: Dietary protein source and manufacturing processes affect macronutrient digestibility, fecal consistency, and presence of fecal clostridium perfringens in adult dogs. J Nutr 2004, 134:2158S-2161S.

62. Minamoto Y, Hooda S, Swanson KS, Suchodolski JS: Feline gastrointestinal microbiota. Anim Heal Res Rev 2012, 13:64-77.

63. Belenguer A, Duncan SH, Calder AG, Holtrop G, Louis P, Lobley GE, Harry J, Flint HJ: Two Routes of Metabolic Cross-Feeding between Bifidobacterium adolescentis and Butyrate-Producing Anaerobes from the Human Gut Two Routes of Metabolic Cross-Feeding between Bifidobacterium adolescentis and Butyrate-Producing Anaerobes from the Human Gut. Appl Environ Microbiol 2006, 72:3593-3599.

64. Kolida S, Tuohy K, Gibson GR: Prebiotic effects of inulin and oligofructose. Br J Nutr 2007, 87:S193-S197.

65. Itoh K, Mitsuoka T, Maejima K, Hiraga C, Nakano K: Comparison of fecal flora of cats based on different housing conditions with special reference to Bifidobacterium. Lab Anim 1984, 18:280-284.

66. Desai AR, Musil KM, Carr AP, Hill JE: Characterization and quantification of feline fecal microbiota using cpn60 sequence-based methods and investigation of animal-to-animal variation in microbial population structure. Vet Microbiol 2009, 137:120-128.

67. Huse SM, Dethlefsen L, Huber J, Mark Welch D, Welch DM, Relman D, Sogin ML: Exploring microbial diversity and taxonomy using SSU rRNA hypervariable tag sequencing. PLOS Genet 2008, 4:2383-2400.

68. Krogius-Kurikka L, Kassinen A, Paulin L, Corander J, Mäkivuokko H, Tuimala J, Palva $A$ : Sequence analysis of percent $G+C$ fraction libraries of human faecal bacterial DNA reveals a high number of Actinobacteria. BMC Microbiol 2009, 9:68.

69. Zentek J, Marquart B, Pietrzak T, Ballèvre O, Rochat F: Dietary effects on bifidobacteria and Clostridium perfringens in the canine intestinal tract. J Anim Physiol Anim Nutr (Berl) 2003, 87:397-407.

70. Endo A, Futagawa-Endo Y, Dicks LMT: Diversity of Lactobacillus and Bifidobacterium in feces of herbivores, omnivores and carnivores. Anaerobe 2010, 16:590-596.

71. King J: Shigella flexneri: A practical review for zoo personnel. Zoo Biol 1998, 17:59-76.

72. Green CE: Infectious Diseases of the Dog and Cat. 4th edition. Philadephia: Saunders; 2012:1376

doi:10.1186/1471-2180-14-43

Cite this article as: Becker et al:: Phylogenetic analysis of faecal microbiota from captive cheetahs reveals underrepresentation of Bacteroidetes and Bifidobacteriaceae. BMC Microbiology 2014 14:43. 